Rev. Biol. Trop., 47(4): 659-676, 1999

www.ucr.ac.cr www.ots.ac.cr www.ots.duke.edu

\title{
Arrecifes coralinos de Bocas del Toro, Panamá: III. Distribución, estructura, diversidad y estado de conservación de los arrecifes de las islas Pastores, Cristóbal, Popa y Cayo Agua
}

\author{
Héctor M. Guzmán \& Carlos A. Guevara \\ Smithsonian Institution, Smithsonian Tropical Research Institute Washington, DC 20560-0580, EEUU. \\ Correo electrónico: guzmanh @ naos.si.edu
}

Recibido 26-VIII-1999. Corregido 1-X-1999. Aceptado 3-X-1999.

\begin{abstract}
Previous studies reported low coral diversity and poor reef development inside the marine protected area of Isla Bastimentos (PNMIB), and it was recommended to widen the survey further east into other areas of the archipelago of Bocas del Toro. This study describes reef distribution, structure and status in 24 reefs along ca. $107 \mathrm{~km}$ of coastline in Pastores, Cristobal, Popa and Agua islands. We define for the first time the main center of diversity or "hot spot" zone for important sessile reef-building organisms. Coral diversity increases to 57 species for the archipelago ( $89 \%$ of Panama), adding up to 64 records for the country. There was also an increase to 29 and 53 species for octocorals and sponges, respectively. For local diversity, $96 \%, 94 \%$ and $97 \%$ of the total number of coral, octocoral and sponge species, respectively, are recorded inside the "hot spot." Based on our results, we recommend the modification of the existing limit of the PNMIB to include reef areas with highest diversity and abundance of threatening species (e.g. Acropora palmata, A. cervicornis), or the creation of a network of marine protected areas within the archipelago.
\end{abstract}

Key words: Biodiversity, coral reefs, marine conservation, marine protected areas, Caribbean, reef assessment, paper park, Bocas del Toro.

La destrucción acelerada de los arrecifes coralinos en el mundo por causas antrópicas y naturales ha sido vista con preocupación por las autoridades responsables del manejo de recursos naturales (Bryant et al. 1998, Wilkinson et al. 1999). Esto en consecuencia ha mejorado la comunicación y el entendimiento entre científicos, administradores de recursos y políticos, aunque todavía existen muchos aspectos relacionados con la conservación de arrecifes que deben ser examinados en conjunto. Estudios sobre la distribución, diversidad y estado actual de conservación de los arrecifes coralinos son necesarios porque proporcionan la información necesaria para una planificación y protección adecuada de la zona costera. Además, estos permiten la identificación de áreas priori- tarias para conservación y mejoran el manejo de áreas protegidas ya existentes.

El Parque Marino Isla Bastimentos (total 13226 ha; 11596 ha son ambientes marinos), en el Archipiélago de Bocas del Toro, es un caso donde pareciera que para el establecimiento de polígonos limítrofes del área marina protegida (ver Alvarado 1987) se consideró más importante el evitar los conflictos sociales, que el mantener la diversidad y los procesos ecológicos esenciales que apoyan los sistemas vivientes y aseguran la utilización sostenible de las especies y el ecosistema (sensu Kelleher y Kenchington 1991). Hasta la fecha se reconoce una diversidad de corales escleractínidos de ca. 54 especies para Bocas del Toro (Guzmán y Guevara 1998b), lo cual representa un 
actual, no considera los sectores arrecifales más importantes del archipiélago, y es por esa razón que se recomendó ampliar los estudios fuera de esa área que permitiera identificar los centros de diversidad vitales de la localidad (sensu Guzmán y Guevara 1998b).

El presente estudio es parte de la serie de cuatro publicaciones que contemplan la descripción total de los arrecifes coralinos e inventario de organismos sésiles asociados a estas comunidades en Bocas del Toro (Guzmán y Guevara 1998a, b). Este tercer estudio incluye únicamente los arrecifes que bordean la zona litoral insular de las islas Pastores, Cristóbal, Popa y Cayo Agua, terminándose así el reconocimiento de la zona insular del archipiélago. Posteriormente, en un último estudio se evaluarán los arrecifes restantes de la región continental comprendidos entre Punta Valiente y el Río Calovévora, límite oriental de la Provincia de Bocas del Toro.

\section{MÉTODOS}

Área de estudio: La Provincia de Bocas del Toro se encuentra localizada al occidente de la República de Panamá entre los $8^{\circ} 30^{\prime}$ y $9^{\circ} 40^{\prime}$ de latitud norte, y entre $81^{\circ} 8^{\prime}$ y los $82^{\circ} 56^{\prime}$

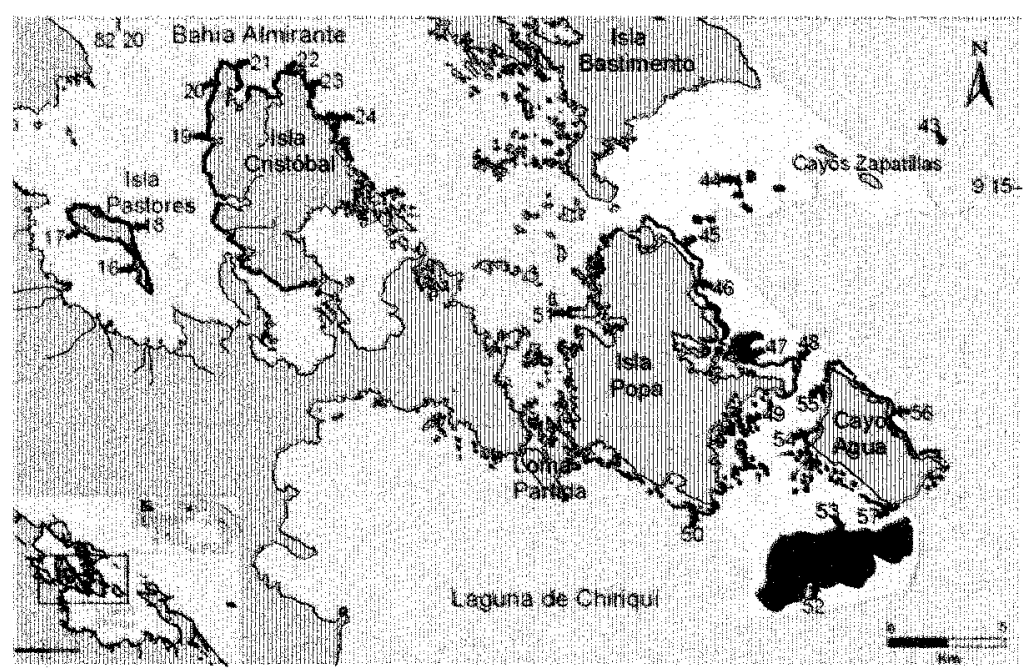

Fig. 1. Bocas del Toro, Panamá (recuadro inferior izquierdo) y detalle de la región principal del presente estudio, islas Pastores, Cristóbal, Popa y Cayo Agua (verde). Se indica la distribución aproximada de los arrecifes coralinos alrededor de las islas (linea y área negra) y el área de mayor diversidad de corales, octocorales y esponjas encontrada en el archipiélago (amarillo). La ubicación geográfica de los 24 arrecifes descritos corresponde al Cuadro 1. de longitud oeste; limita por el norte con el Mar Caribe, al sur con la Provincia de Chiriquí, al este con la Provincia de Veraguas, y al oeste con la República de Costa Rica. La provincia tiene una superficie de ca. $8917 \mathrm{~km}^{2}$ (Rodríguez et al. 1993). Información detallada sobre el clima, geología y orografía está disponible (ver Gordon 1982, Anónimo 1988, Rodríguez et al. 1993, Collins et al. 1996, Coates y Jackson 1998) y resumida en (Greb et al. 1996, Guzmán y Guevara 1998a).

Reconocimiento de arrecifes y muestreo: Se hizo un reconocimiento detallado en bote de la zona costera insular mencionada arriba (Fig. 1) describiéndose la distribución y estado de conservación de los arrecifes coralinos, y a su vez, se observó las condiciones actuales de áreas de bosques deforestadas cercanas a la costa. Durante este recorrido se identificaron los arrecifes que serían usados posteriormente para la descripción detallada. Además, se realizaron reconocimientos puntuados en parches coralinos de diversos tamaños localizados entre las distintas islas y referenciados por cartas náuticas (Defense Mapping Agency; 28041, 28042, 28045). Todos los nombres que se emplean en el presente estudio para caracterizar la localización de cada arrecife, se obtuvieron principalmente de mapas (escala 1:50 000) publicados por el Instituto Geográfico Nacional "Tommy Guardia" en 1991. La presentación en la Fig. 1 de la distribución de arrecifes coralinos se considera aproximada debido a su escala. 


\section{CUADRO 1}

Localización de los 24 arrecifes coralinos estudiados en las Islas Pastores, Cristóbal, Popa, y Cayo Agua, Bocas del Toro, Panamá (ver Fig. 1), y la profundidad máxima de desarrollo arrecifal (metros).

\begin{tabular}{|c|c|c|}
\hline $\mathrm{N}^{\circ}$ & Latitud/Longitud & Localidad \\
\hline 16 & $9^{\circ} 13^{\prime} 11^{\prime \prime} \mathrm{N} / 82^{\circ} 19^{\prime} 31 " \mathrm{~W}$ & Cayo Roldán Noroeste \\
\hline 17 & $9^{\circ} 14^{\prime} 05^{\prime \prime} \mathrm{N} / 82^{\circ} 20^{\prime} 44^{\prime \prime} \mathrm{W}$ & Punta Ford \\
\hline 18 & $9^{\circ} 14^{\prime} 14^{\prime \prime} \mathrm{N} / 82^{\circ} 19^{\prime} 47^{\prime \prime} \mathrm{W}$ & Punta Vergayo \\
\hline 19 & $9^{\circ} 16^{\prime} 19^{\prime \prime} \mathrm{N} / 82^{\circ} 17^{\prime} 55^{\prime \prime} \mathrm{W}$ & Isla Cristóbal Oeste \\
\hline 20 & $9^{\circ} 17^{\prime} 23^{\prime \prime N} / 82^{\circ} 17^{\prime} 44^{\prime \prime} \mathrm{W}$ & Isla Cristóbal Noroeste \\
\hline 21 & $9^{\circ} 17^{\prime} 54^{\prime \prime} \mathrm{N} / 82^{\circ} 17^{\prime} 22^{\prime \prime} \mathrm{W}$ & Punta Juan \\
\hline 22 & $9^{\circ} 17^{\prime} 46^{\prime \prime N} / 82^{\circ} 16^{\prime} 04^{\prime \prime} \mathrm{W}$ & Punta Coco \\
\hline 23 & $9^{\circ} 17^{\prime} 22^{\prime \prime} \mathrm{N} / 82^{\circ} 15^{\prime} 32^{\prime \prime} \mathrm{W}$ & Isla Cristóbal Nordeste \\
\hline 24 & $9^{\circ} 16^{\prime} 47^{\prime \prime N} / 82^{\circ} 14^{\prime} 48^{\prime \prime} \mathrm{W}$ & Isla Cristóbal Sureste \\
\hline 43 & $9^{\circ} 15^{\prime} 37^{\prime \prime} \mathrm{N} / 82^{\circ} 00^{\prime} 42^{\prime \prime} \mathrm{W}$ & Bajo Zapatilla Norte \\
\hline 44 & $9^{\circ} 15^{\prime} 05^{\prime \prime N} / 82^{\circ} 05^{\prime} 06^{\prime \prime} \mathrm{W}$ & Bajo Laguna Zapatilla \\
\hline 45 & $9^{\circ} 14^{\prime} 03^{\prime \prime N} / 82^{\circ} 06^{\prime} 41^{\prime \prime} \mathrm{W}$ & Isla Popa-Cayo Deer \\
\hline 46 & $9^{\circ} 13^{\prime} 15^{\prime \prime} \mathrm{N} / 82^{\circ} 06^{\prime} 07^{\prime \prime} \mathrm{W}$ & Isla Popa Norte \\
\hline 47 & $9^{\circ} 11^{\prime} 18^{\prime \prime} \mathrm{N} / 82^{\circ} 05^{\prime} 02^{\prime \prime} \mathrm{W}$ & Isla Popa-Buena Vista \\
\hline 48 & $9^{\circ} 11^{\prime} 08^{\prime \prime} \mathrm{N} / 82^{\circ} 04^{\prime} 05^{\prime \prime} \mathrm{W}$ & Isla Popa-Isla Tigre \\
\hline 49 & $9^{\circ} 09^{\prime} 30^{\prime \prime} \mathrm{N} / 82^{\circ} 05^{\prime} 10^{\prime \prime} \mathrm{W}$ & Isla Popa-La Mina \\
\hline 50 & $9^{\circ} 07^{\prime} 35^{\prime \prime} \mathrm{N} / 82^{\circ} 06^{\prime} 30^{\prime \prime} \mathrm{W}$ & Isla Popa-Punta Laurel \\
\hline 51 & $9^{\circ} 12^{\prime} 13^{\prime \prime} \mathrm{N} / 82^{\circ} 0923^{\prime \prime} \mathrm{W}$ & Isla Popa-Cayo Wilson \\
\hline 52 & $9^{\circ} 06^{\prime} 28^{\prime \prime N} / 82^{\circ} 02^{\prime} 49^{\prime \prime} \mathrm{W}$ & Cayo Agua-Bajo Norte \\
\hline 53 & $9^{\circ} 05^{\prime} 13^{\prime \prime} \mathrm{N} / 82^{\circ} 03^{\prime} 38^{\prime \prime} \mathrm{W}$ & Cayo Agua-Bajo Sur \\
\hline 54 & $9^{\circ} 09^{\prime} 39^{\prime \prime} \mathrm{N} / 82^{\circ} 03^{\prime} 52^{\prime \prime} \mathrm{W}$ & Cayo Agua Suroeste \\
\hline 55 & $9^{\circ} 10^{\prime} 24^{\prime \prime} \mathrm{N} / 82^{\circ} 0320^{\prime \prime} \mathrm{W}$ & Cayo Agua-Punta Norte \\
\hline 56 & $9^{\circ} 09^{\prime} 46^{\prime \prime} \mathrm{N} / 82^{\circ} 01^{\prime} 42^{\prime \prime} \mathrm{W}$ & Cayo Agua-Punta Níspero \\
\hline 57 & $9^{\circ} 07^{\prime} 50 " \mathrm{~N} / 82^{\circ} 02^{\prime} 05^{\prime \prime} \mathrm{W}$ & Cayo Agua-Punta Roca Roja \\
\hline
\end{tabular}

El estudio comprende tres aspectos básicos para la evaluación de los arrecifes de coral: 1) distribución de los arrecifes coralinos; 2) inventario sobre la diversidad (número de especies) de corales y algunos organismos principales asociados al arrecife (plantas y animales); y 3 ) zonación, cobertura y abundancia relativa de corales y principales organismos sésiles en arrecifes de la región (adaptado de Loya 1972, Sullivan y Chiappone 1992, Sullivan et al. 1994). Esto permitirá evaluar el estado actual del ecosistema en términos de cobertura y diversidad, y proyectar así las áreas naturales vulnerables a programas de desarrollo presentes y futuros en la región. El presente estudio sirve de línea de base a futuras investigaciones que se realizarán en la región. Detalles de la metodología de muestreo han sido descritos en publicaciones previas (Guzmán y Guevara 1998a), pero se resumen a continuación:
(1) Durante 80 min se hizo un reconocimiento completo del arrecife desde la parte más profunda hasta la somera, anotando la composición de especies y diversidad de los organismos sésiles y taxones más comunes, incluidos los corales escleractínidos, octocoralarios, esponjas, algas, y otros. Las especies de organismos sésiles fueron clasificados usando publicaciones selectas para esponjas, anémonas, octocorales y corales escleractínidos, cuyas citas se proporcionaron en los primeros trabajos (Guzmán y Guevara 1998a, b).

(2) Se escogieron al azar tres transectos de $5 \times 1 \mathrm{~m}$ cada uno y separados entre si por la misma distancia, paralelos a la costa y en cada una de las tres profundidades seleccionadas para describir visualmente la cobertura de organismos en cada arrecifes ( $1 \mathrm{~m}, 3 \mathrm{~m}$ y $10 \mathrm{~m}$ ), para un total de $45 \mathrm{~m}^{2}$ por arrecife. Además, se 
estimó el número de individuos juveniles o reclutas $/ \mathrm{m}^{2}$ de especies de coral presentes en los transectos. La numeración asignada a los arrecifes (Fig. 1) corresponde a la base de datos del Instituto Smithsonian de Investigaciones Tropicales, la cual contiene otros arrecifes descritos anteriormente para la región (ver Guzmán y Guevara 1998a, b).

\section{RESULTADOS}

En conjunto, se encontró una diversidad total de especies entre las distintas islas de 52 corales escleractínidos (con tres nuevos registros para Panamá), 25 octocorales y 50 esponjas (Cuadros 2-4). Un análisis de grupo donde se compara dicha diversidad entre los 24 arrecifes descritos y basado en el total de especies para estos tres taxones, separa la región en dos grandes grupos (Fig. 2). Este agrupamiento destaca una zona de mayor diversidad (grupo inferior) en estos organismos sésiles hacia la región oriental del área de estudio (Fig. 1, zona amarilla).

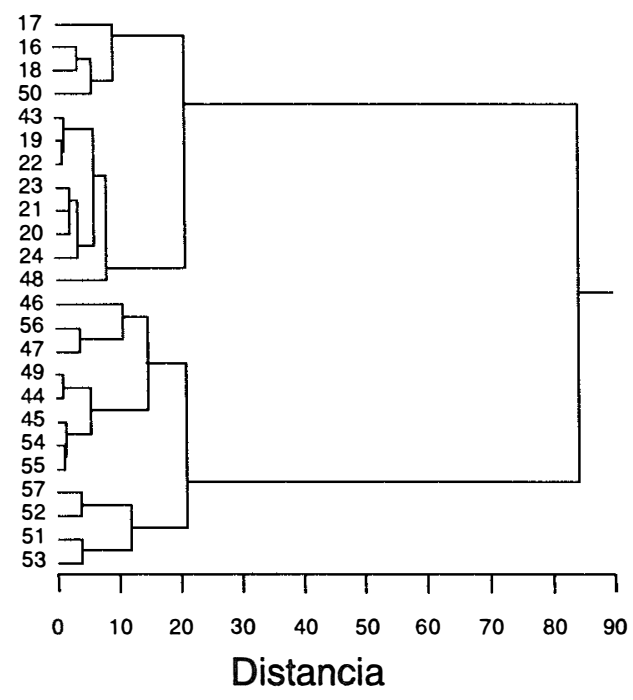

Fig. 2. Análisis de grupos comparando la diversidad total de especies de los tres táxones más importantes estudiados (escleractínidos, octocorales y esponjas) entre los 24 arrecifes descritos para esta parte de la región insular del archipiélago de Bocas del Toro. El dendrograma se basa en distancias euclidianas, amalgamados por el método de Ward.
En la Fig. 3 se presenta la cobertura de los diferentes taxones agrupados por profundidad y en conjunto para cada isla. Se encontró una gradiente Oeste-Sureste en el área de estudio con una disminución general de la cobertura de coral vivo total y por profundidad, siendo mayor en Isla Pastores y menor hacia Cayo Agua. Esta tendencia se explica por la alta densidad que representa el coral Porites furcata. Igual comportamiento se observa para las esponjas, con una disminución notable hacia aguas más profundas. La cobertura de macroalgas es superior al $30 \%$ en todas las islas en casi todas las profundidades, mientras que las algas coralinas incrustantes presentan una tendencia contraria y superan el $20 \%$ en varias de las islas. Este último grupo en particular se hace notable en aguas profundas. Los octocorales también parecen aumentar con la profundidad y con tendencia contraria a los corales escleractínidos, aunque por lo general este aumento es debido a la abundancia de Eythropodium caribaeorum (Fig. 3).

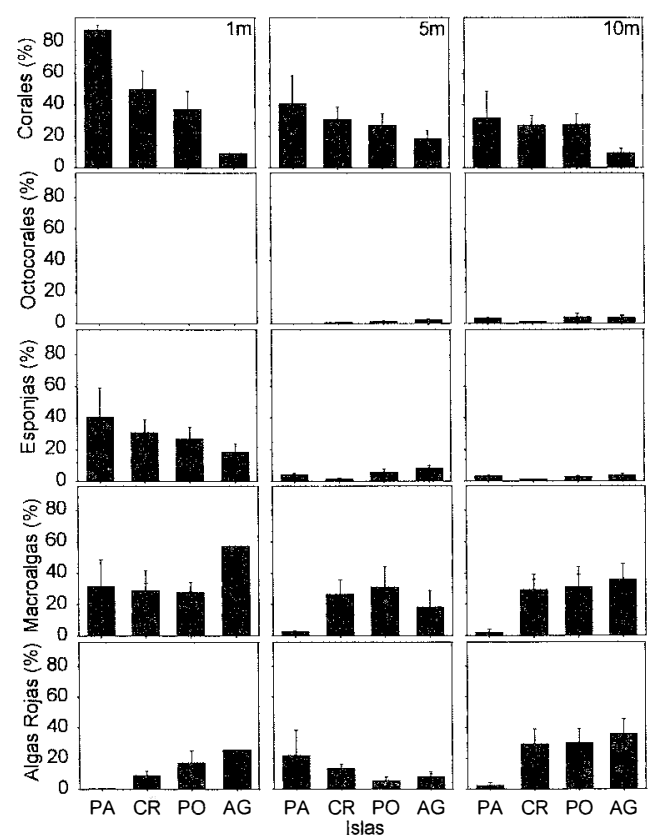

Fig. 3. Porcentaje promedio y error estándar para la distribución vertical de cobertura relativa de escleractínidos, octocorales, esponjas, macroalgas, y algas rojas coralinas por $\mathrm{m}^{2}$, para cada profundidad y agrupados por los arrecifes descritos en cada isla, en Bocas del Toro, Panamá. Las letras en eje inferior indican las Islas Pastores (PA), Cristóbal (CR), Popa (PO) y Cayo Agua (AG). 
A continuación se describen con más detalle las características de los arrecifes para cada isla, incluyendo observaciones sobre los ecosistemas terrestres que pueden afectar los arrecifes que las rodean.

Isla Pastores: Se describieron tres arrecifes en $11.3 \mathrm{~km}$ de costa (Fig. 1), encontrándose una cobertura de corales vivos promedio de ca. $53 \%$ e inferiores al $5 \%$ para octocorales, esponjas y macroalgas. Los arrecifes están bien desarrollados hasta los $20 \mathrm{~m}$ de profundidad, y en los ambientes someros están rodeados por una barrera coralina sólida y contínua construida por el coral dedo Porites furcata (sensu Guzmán y Guevara 1998a), la cual crea un ambiente lagunar dominado por pastos marinos. El talud arrecifal está dominado por el coral lechuga Agaricia tenuifolia hasta los $6 \mathrm{~m}$, mezclado en algunas áreas con
$P$. furcata y esponjas. Hacia el talud inferior y la base, los arrecifes están dominados por el coral masivo Siderastrea siderea entre amplios parches de arena (Fig. 4). No se observaron corales reclutas o individuos juveniles abundantes en estos arrecifes (Fig. 5). La diversidad de corales es relativamente baja (1318 especies), al igual que para octocorales y esponjas (Cuadros 2-4).

En términos generales los arrecifes se encuentran en buen estado de conservación (por la cobertura) a pesar de que existe una extensa ganadería en el sector sur de la isla entre Punta Maya y Punta Ford, y regiones amplias deforestadas al norte, entre Punta Maya y Punta Vergayo. El bosque de manglar que rodea la isla está bien conservado en el sector norte y sur, en particular hacia el sureste en los cayos García y Roldán.

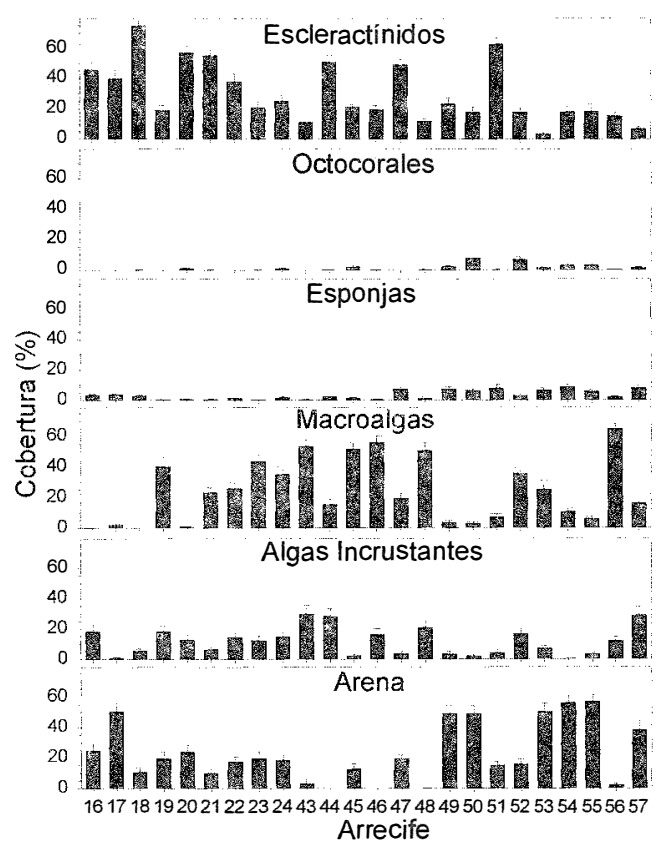

Fig. 4. Porcentaje promedio total y error estándar para la cobertura relativa de coral vivo escleractínidos, octocorales, esponjas, macroalgas, algas rojas coralinas, y sustrato arenoso por $\mathrm{m}^{2}$, para cada arrecife descrito, en Bocas del Toro, Panamá. Números en eje inferiorindican los 24 arrecifes estudiados.

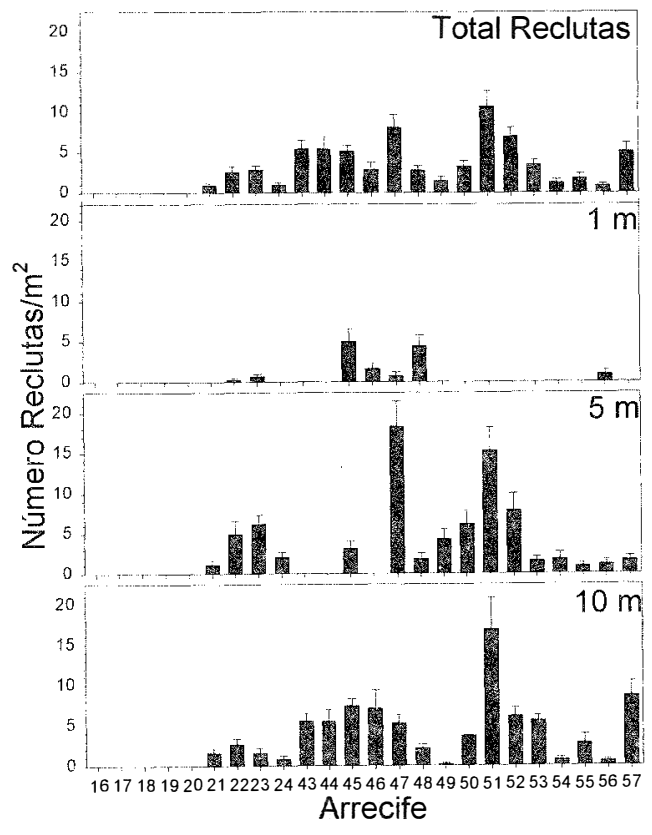

Fig. 5. Número total por $\mathrm{m}^{2}$ de corales escleractínidos reclutas (individuos de tamaño inferior a $4 \mathrm{~cm}$ ) para cada arrecife descrito y por las tres profundidades. 
Lista de corales escleractínios encontraa 's hasta una profindidad de 25 m en cada uno de los 24 arrecifes estudiados en las Islas Pastores, Cristóbal, Popa, y Cayo Agua, Provincia de Bocas del Toro, Panamá. Especie presente ( $x$ ).

Especies

$\begin{array}{llllllllllllllllllllllll}16 & 17 & 18 & 19 & 20 & 21 & 22 & 23 & 24 & 43 & 44 & 45 & 46 & 47 & 48 & 49 & 50 & 51 & 52 & 53 & 54 & 55 & 56 & 57\end{array}$

Acropora cervicornis

Acropora palmata

Agaricia agaricites

Agaricia danai

Agaricıa fragilis

Agaricia humilis

Agaricia lamarcki

Agaricia purpurea

Agaricia tenuifolia

Agaricia undata

Colpophyllia amaranthus

Colpophyllia breviserialis

Colpophyllia natans

Dichocoenia stockes

Diploria clivosa

Diploria labyrinthiformis

Diploria strigosa

Eusmilia fastigiata

Favia fragum

Isophyllastrea rigida

Isophyllia sinuosa

Leptoseris cucullata

Madracis decactis

Madracis mirabilis

Manicina areolata

Manicina mayori

Meandrina meandrite

Millepora alcicornis

Millepora complanata

Montastraea annularis

Montastraea cavernosa

Montastraea faveolata

Montastraea franksi

$\begin{array}{lll}x & x & x\end{array}$

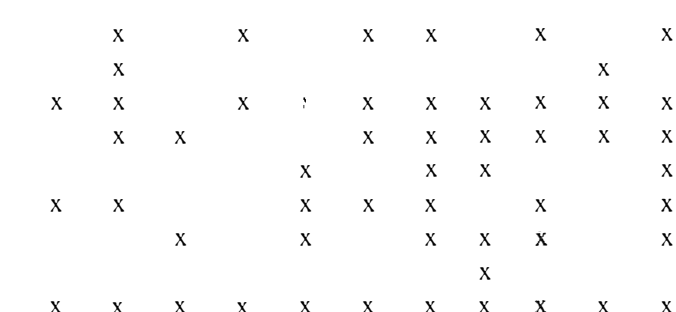

$\begin{array}{llllllllllllllllllll}x & x & x & x & x & x & x & x\end{array}$

$x$
$x$

$\begin{array}{lll}x & x & x \\ x & & x\end{array}$

$\begin{array}{lll}x & x & x \\ x & x & x\end{array}$

$x \quad x$

$\begin{array}{lll} & x & x \\ x & x & x \\ & x & x\end{array}$

$\mathrm{x}$

$$
\mathrm{x}
$$

(n)

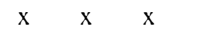

$x \quad x$

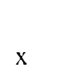


CUADRO 2 (Contaución)

Lisia de corales escleractinios encoitrados hasta ana profindidad de 25 m en cada uno de los 24 arrecifes estudiados en las Islas Pastores, Cristóbal, Popa, y Cavor Agua. Provincia de Bocas del Toro, Panamá. Especie presente $(x)$

Especies

Mussa angulosu

Mycetophyllia aliciae

Mycetophyllica danaan

Mycetophyllia ferox

Mycetophyllia lamarckiana

Mycetophyllici reesi

Oculina diffusa

Porites astreoides

Porites colonensis

Pories divaricata

Porites furcuta

Porites porites

Scolymia cubensis

Scolymia lacera

Siderasirea radian.

Siderustrou siderea

Solenastrea bournoni

Solenastrea hyades

Stephanocoenia interseptu $x$

Total
$16 \quad 17 \quad 18 \quad 19 \quad 20$

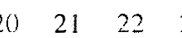

24

$344 \quad 45$

$46 \quad 47$

$48 \quad 49$

$50 \quad 51$

$\begin{array}{lllllll}1 & 52 & 53 & 54 & 55 & 56 & 57\end{array}$ 
Lista de especies de octocorales, anémonas $\left(^{*}\right)$, zoántidos $\left(^{* *}\right)$, y coral negro $\left(^{* * *}\right)$ encontrados hasta una profundidad de 25 m en los 24 arrecifes estudiados en las Islas Pastores, Cristóbal, Popa y Cayo Agua, Bocas del Toro, Panamá. Especie presente (X).

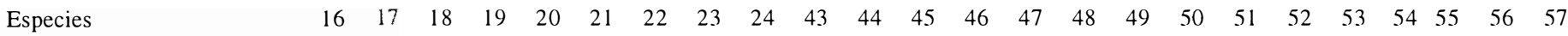

Briareum asbestinum

Erythropodium caribaeorum

Eunicea calyculata

Eunicea fusca

Eunicea mammosa

Eunicea succinea

Eunicea tourneforti

Eunicea sp.

Gorgonia flabellum

Gorgonia ventalina

Gorgonia ventalina

Muricea muricata

Muricea pinnata

Plexaura kuna

Plexaura flexuosa

Plexaura homomalla

Plexaurella nutans

Plexaurella sp

Pseudoplexaura sp

Pseudopterogorgia acerosa

Pseudopterogorgia americana

Pseudopterogorgia bipinnata

Pterogorgia anceps

Pterogorgia citrina

Pterogorgia guadalupensis

$\begin{array}{llllllllllllllllllllllllll}\text { Total de especies de octocorales } & 1 & 0 & 1 & 2 & 5 & 2 & 2 & 2 & 5 & 2 & 12 & 15 & 16 & 9 & 1 & 11 & 6 & 5 & 15 & 2 & 15 & 14 & 10 & 12\end{array}$

Bartholomea lucida*

Condylactis gigantea*

Epicystis crucifer

Lebrunia dana**

Stichodactyla helianthus

Zoanthus pulchellus**

Palythoa caribaeorum**

Cirrhipathes leutkeni***

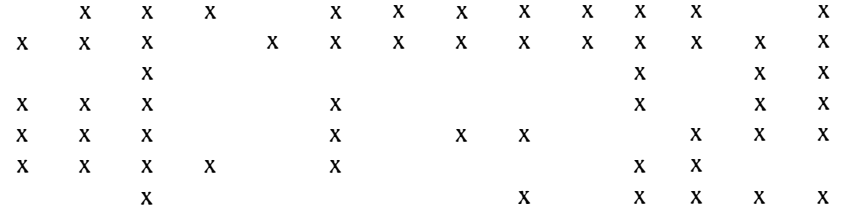

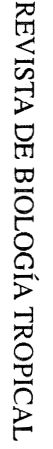


Lista de especies de esponjas encontradas hasta una profundidad de $25 \mathrm{~m}$ en los 24 arrecifes estudiados en las Islas Pastores, Cristobal, Popa, y Cayo Agua, Provincia de Bocas del Toro, Panamá. Especie presente (X).

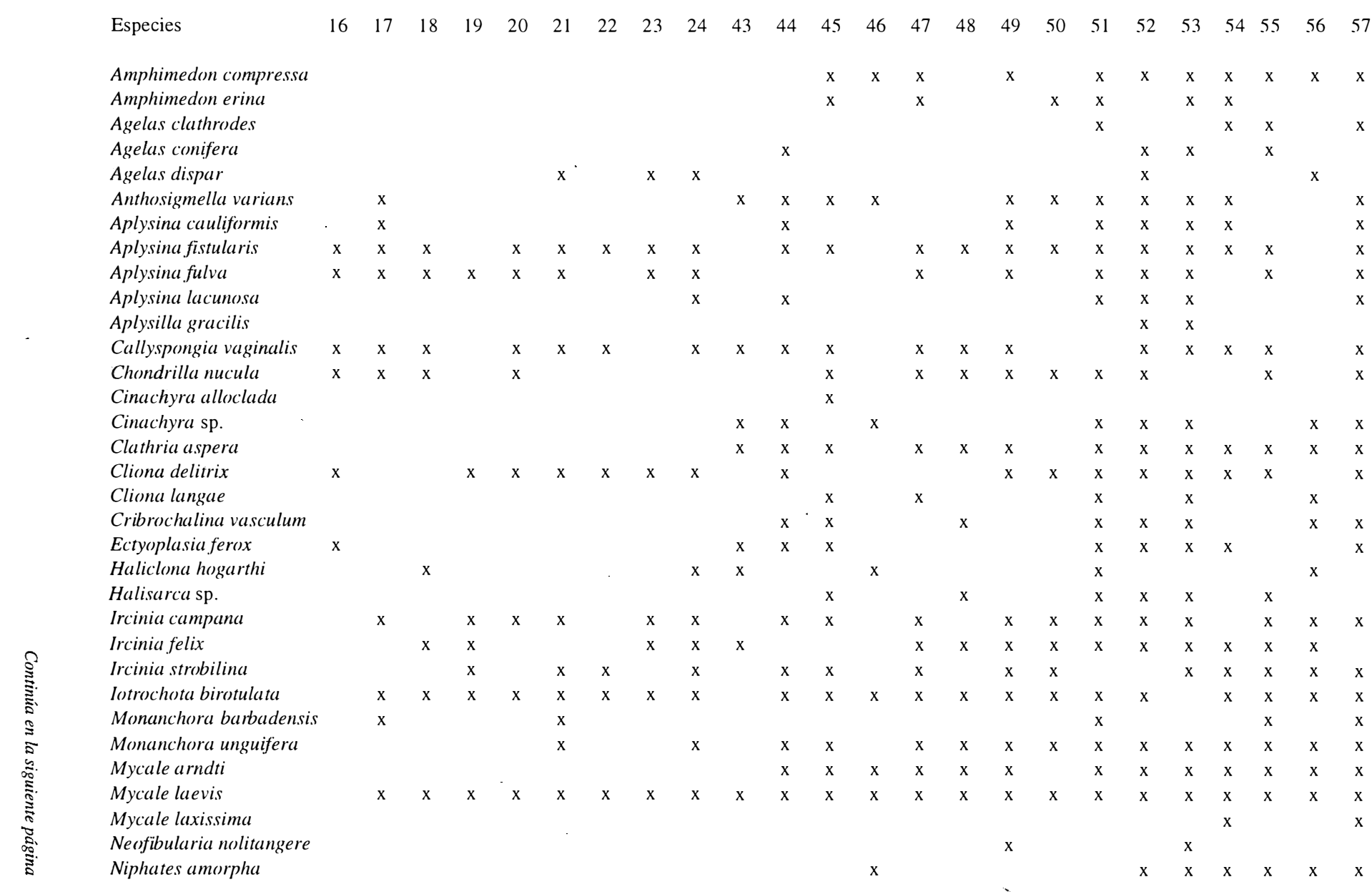


Lista de especies de esponjas encontradas hasta una profundidad de 25 m en los 24 arrecifes estudiados en las Islas Pastores, Cristobal, Popa, y Cayo Agua, Provincia de Bocas del Toro, Panamá. Especie presente (X).

\begin{tabular}{|c|c|c|c|c|c|c|c|c|c|c|c|c|c|c|c|c|c|c|c|c|c|c|c|}
\hline Especies & 16 & 17 & 18 & 19 & 20 & 21 & 22 & 23 & 24 & 43 & 44 & 45 & 46 & 47 & 48 & 49 & 50 & 51 & 52 & 53 & 54 & 55 & 56 \\
\hline Niphates erecta & & & & & & & & & & & $\mathrm{x}$ & $\mathrm{x}$ & $\mathrm{x}$ & $\mathrm{x}$ & $\mathrm{x}$ & $x$ & $\mathrm{x}$ & $\mathrm{x}$ & $\mathrm{x}$ & $x$ & $\mathrm{x}$ & $\mathrm{x}$ & $\mathrm{x}$ \\
\hline Niphates ramosa & & & & & & & & & & & & $\mathrm{x}$ & & $\mathrm{x}$ & & $x$ & & $\mathrm{x}$ & & & $x$ & & $\mathrm{x}$ \\
\hline Oceanapia bartschi & & & $\mathrm{x}$ & & $\mathrm{x}$ & $\mathrm{x}$ & $\mathrm{x}$ & $\mathrm{x}$ & $\mathrm{x}$ & & & $\mathrm{x}$ & & $x$ & & $x$ & & $\mathrm{x}$ & $\mathrm{x}$ & $\mathrm{x}$ & $\mathrm{x}$ & $\mathrm{x}$ & \\
\hline Pachypellina podatypa & & & & & & & & & & & & & & & $\mathrm{x}$ & & & & $\mathrm{x}$ & & & & \\
\hline Plakortis angulospiculatus & & & & & & & & & & & & & & & & $\mathrm{x}$ & & $x$ & & $\mathrm{x}$ & & $\mathrm{x}$ & $\mathrm{x}$ \\
\hline Plakortis halichondroides & & & & & & & & & & & & $\mathrm{x}$ & & $\mathrm{x}$ & & & $\mathrm{x}$ & $\mathrm{x}$ & & & $\mathrm{x}$ & $\mathrm{x}$ & $\mathrm{x}$ \\
\hline Pseudoceratina crassa & $\mathrm{x}$ & $\mathrm{x}$ & $\mathrm{x}$ & $\mathrm{x}$ & $\mathrm{x}$ & $\mathrm{x}$ & $x$ & $x$ & $\mathrm{x}$ & $\mathrm{x}$ & $\mathrm{x}$ & & & & & & $\mathrm{x}$ & $\mathrm{x}$ & $\mathrm{x}$ & $\mathrm{x}$ & $\mathrm{x}$ & $\mathrm{x}$ & \\
\hline Pseudaxinella lunaecharta & & $\mathrm{x}$ & & $x$ & $\mathrm{x}$ & & & & & $\mathrm{x}$ & $\mathrm{x}$ & $\mathrm{x}$ & & $\mathrm{x}$ & & & & $\mathrm{x}$ & $\mathrm{x}$ & & $\mathrm{x}$ & & $\mathrm{x}$ \\
\hline Ptilocaulis walpersi & & & & & & & & & & & $x$ & & & & & & & $\mathrm{x}$ & $\mathrm{x}$ & $\mathrm{x}$ & & & $\mathrm{x}$ \\
\hline Siphonodictyon corallipha & gum & & & & & & & & & & & $\mathrm{x}$ & & & & $\mathrm{x}$ & & & $\mathrm{x}$ & & & & \\
\hline Spheciospongia vesparium & & & & & & & & & & & & $\mathrm{x}$ & & & & $\mathrm{x}$ & & $\mathrm{x}$ & $\mathrm{x}$ & $\mathrm{x}$ & & $\mathrm{x}$ & \\
\hline Spirastrella coccinea & & & & & & & & & & & $\mathrm{x}$ & & & $\mathrm{x}$ & $\mathrm{x}$ & & & $\mathrm{x}$ & $\mathrm{x}$ & $\mathrm{x}$ & $\mathrm{x}$ & $\mathrm{x}$ & $\mathrm{x}$ \\
\hline Ulosa ruetzleri & & & & & & & & & & & & $x$ & & & $\mathrm{x}$ & & & & $\mathrm{x}$ & $\mathrm{x}$ & & & \\
\hline Verongula rigida & & & & & & & & & & & & & & & & & & & & & & $\mathrm{x}$ & \\
\hline Xetospongia muta & & & & & & & & & & & $\mathrm{x}$ & $\mathrm{x}$ & & $\mathrm{x}$ & & $\mathrm{x}$ & $\mathrm{x}$ & $\mathrm{x}$ & & & $\mathrm{x}$ & $\mathrm{x}$ & \\
\hline etospongia proxima & & & & & & & & & & & & & & & & & & & $\mathrm{x}$ & & & & \\
\hline etospongia rosariensis & & & & & & & & & & & & & & & & & & $\mathrm{x}$ & & & $\mathrm{x}$ & & \\
\hline otal & 7 & 12 & 10 & 9 & 11 & 13 & 8 & 10 & 15 & 10 & 24 & 26 & & 22 & 16 & 23 & 15 & 37 & 34 & 33 & 27 & 28 & 22 \\
\hline
\end{tabular}


Isla Cristóbal: Representa ca. 38.9 km de costa, describiéndose en esta isla seis arrecifes en total (Fig. 1). Los arrecifes forman una barrera somera de P. furcata con buen desarrollo y un talud extenso de Agaricia spp. con una gradiente leve hasta los $20 \mathrm{~m}$ de profundidad en el lado oeste, desde Punta Rodríguez hasta Punta Juan y solo interrumpida por dos ensenadas pequeñas con poblados. Los corales de profundidad, A. lamarcki y en particular A. undata, abundan en estos arrecifes y no en otras localidades (Cuadro 2). Entre Punta Juan y Punta Coco, al norte de la isla, la barrera se interrumpe por dos amplias ensenadas con bastantes sedimentos aportados por la Quebrada Botabite. Luego continúa formando extensas áreas con barreras y parches arrecifales hasta llegar a los numerosos cayos de manglar del sureste, que sirven de entrada a la Laguna de Bocatorito (Fig. 1). En el sector de Bocatorito, al suroeste de Isla Cristóbal y en la ensenada de la Laguna de Tierra Oscura se encuentran parches someros de $P$. furcata y pequeñas barreras interrumpidas y con poco desarrollo formadas por colonias dispersas de Agaricia tenuifolia, Colpophyllia spp. y Siderastrea siderea. Entre los arrecifes descritos alrededor de la isla (19-24) se encontró una cobertura de coral alta $(>30 \%)$ en todos los arrecifes al extremo norte de esta (Fig. 4). Los octocorales y esponjas no variaron mucho, mientras que las macroalgas oscilaron entre 20 y $45 \%$. Las algas incrustantes por lo general presentaron un promedio de $30 \%$, con sectores completamente cubiertos por el alga no incrustante Amphiroa rigida var. antillana. Se observaron patrones de reclutamiento de corales moderados, en algunos casos superiores a 5 individuos $/ \mathrm{m}^{2}$ a profundidades intermedias (Fig. 5). La diversidad de corales observada fue moderada oscilando entre 23 y 28 especies, siempre mayor en los arrecifes al este de la isla (Cuadro 2). Los ámbitos de diversidad fueron bajo en octocorales y moderado en esponjas con 2-5 y 8-15 especies, respectivamente (Cuadros 3 y 4 ).

En la Isla Cristóbal se observa un alto deterioro de los ambientes terrestres, particularmente de los bosques en las montañas del sec- tor sur-suroeste de la isla, incluyendo muchas áreas de manglar. Todo el sector sur de Isla Cristóbal está afectado por alta sedimentación proveniente de ríos del continente y quebradas locales. Los ríos que nacen en esta isla acarrean altas concentraciones de sedimentos arcillosos hacia los arrecifes.

Isla Popa: Se describieron ocho arrecifes en ca. $37.3 \mathrm{~km}$ de zona costera, incluyendo un arrecife profundo entre Cayos Zapatillas y la isla (Fig. 1). Toda la zona norte de la isla está rodeada por una barrera arrecifal que forma amplias lagunas con pastos marinos. Estos arrecifes están bien desarrollados pero son poco profundos. La mayor parte de esta barrera norte estaba construida por el coral Acropora palmata, poco abundante en estos momentos. La barrera se hace más discontinua hacia los sectores este y oeste alrededor de la isla y está formada por Porites furcata, al igual que en otras localidades. El sector sur de la isla esta formado principalmente por parches someros de esta última especie y Agaricia tenuifolia. En todo el sector norte se encuentran parches aislados de A. palmata y A. cervicornis en aguas someras, mezclado con colonias grandes del coral Siderastrea siderea.

La cobertura de coral es relativamente baja en la mayoría de los arrecifes ( $<20 \%)$, excepto los arrecifes semi-protegidos 44,47 y 51 que presentan coberturas superiores al $45 \%$ (Fig. 4). Sin embargo, los arrecifes profundos y parches someros que se encuentran entre el nordeste de la isla y los Cayos Zapatillas (similar al arrecife 44) tienen altas coberturas y están compuestos por importantes especies constructoras de arrecifes como Montastraea faveolata, A. palmata y A. cervicomis. La cobertura alta de macroalgas coincide por lo general con estos arrecifes menos densos en corales y por lo general expuestos directamente al oleaje, y no varió entre profundidades (Fig. 3). Hacia el canal que separa la isladel Cayo Agua se encuentra la mayor cobertura de esponjas y octocorales (Fig. 4). El reclutamiento promedio fue de 5 individuos $/ \mathrm{m}^{2}$, pero se duplicó en los arrecifes 47 y 51, siendo este mayor en las áreas arrecifales más profundas (Fig. 5). La diversidad de corales es moderada a 
alta, oscilando entre 19 y 35 especies y sin un patrón de distribución definido alrededor de la isla (Cuadro 2). Igual resulta la diversidad de octocorales (ámbito de 1-16 especies) (Cuadro 3 ), aunque se observa un patrón de distribución con mayor diversidad hacia el sector norte de la isla y los Cayos Zapatillas (Fig. 1). La diversidad de esponjas (9-37 especies) y su distribución es amplia alrededor de la isla (Cuadro 4). $\mathrm{Al}$ suroeste de Isla Popa, existen numerosos bajos coralinos someros de tamaños que oscilan entre 200 y $5000 \mathrm{~m}^{2}$ que fueron visitados pero no descritos y en los cuales se alberga la mayor diversidad de esponjas y octocorales.

Isla Popa se encuentra deforestada en todas las áreas cercanas a asentamientos indígenas, particularmente los sectores oeste y nor' deste (Isla Tigre). Por lo general, los manglares franjeantes e islotes están bien conservado alrededor de la isla; la tala es muy localizada a la entrada de poblados. El arrecife 51 a pesar de estar cercano a áreas deforestadas, presentó una de las coberturas de coral más altas y mejor desarrollo arrecifal de todo el estudio, al igual que mayor densidad de reclutas a profundidades superiores a los $4 \mathrm{~m}$, posiblemente debido al flujo de corrientes fuertes de distintas direcciones que convergen en ese sector.

Cayo Agua: Se describieron seis arrecifes en ca. $20 \mathrm{~km}$ de costa, incluyendo un bajo arrecifal extenso (ca. $22 \mathrm{~km}^{2}$ ) localizado al sur del cayo (Fig.1). El cayo está rodeado por una barrera interrumpida de coral en el lado norte, con pequeñas ensenadas donde se encuentran parches amplios de Acropora palmata, entre Punta Níspero y Punta Tiburón. Hacia el sector este y sur del cayo, no existe una verdadera barrera, pero se forma un sistema lagunar somero donde sobresalen extensos y numerosos parches de $P$. furcata entremezclados con pastos marinos, en particular el sector entre Punta Limón y Punta Piedra Roja. Los arrecifes bordean ambos lados pero a partir de los $4 \mathrm{~m}$ de profundidad, donde pueden dominar áreas de esponjas y octocorales. Sin embargo, los arrecifes que rodean el bajo al sur del cayo presentan un impresionante grado de desarrollo hasta los $25 \mathrm{~m}$ de profundidad, pero estos están com- pletamente muertos en las áreas más profundas, donde se observa una estructura que fue construida por Montastraea spp., Madracis spp. y Siderastrea siderea. Las áreas someras de este bajo están dominadas por Millepora spp. mezclado con $P$. furcata.

Se observó la cobertura de corales escleractínidos más baja del archipiélago, ca. $15 \%$, pero la diversidad más alta, que osciló entre 27 y 38 especies. Mientras que los octocorales se presentaron con promedios altos de cobertura relativa, $3.5 \%$, y diversidad también alta, entre 2 y 15 especies. Las esponjas alrededor de Cayo Agua alcanzan la máxima cobertura (ca. $4 \%$ ) y diversidad (22-37 especies) en muchos de los arrecifes en comparación con el resto de los estudiados (Fig. 4, Cuadros 2-4). Las macroalgas y algas incrustantes oscilaron àmpliamente en su cobertura (5-60\% y $0.5-25 \%$, respectivamente), encontrándose el arrecife de mayor porcentaje en el extremo nordeste del cayo (Fig. 4). El número de reclutas es ba jo en promedio (ca. 3 colonias $/ \mathrm{m}^{2}$ ) y se observa un patrón de reclutamiento mayor entre el sector sur del cayo y el bajo extenso, principalmente en aguas profundas (Fig. 5).

La deforestación en Cayo Agua es similar a la observada para Isla Popa, siendo particularmente extensa en el sector oeste-suroeste entre Punta Norte y Punta Limón, en áreas donde se encuentran poblados indígenas. En otras áreas alrededor del cayo, la deforestación es puntuada y en pequeñas parcelas.

Bajos de Cayos Zapatillas: En reconocimientos anteriores se describieron los arrecifes de Cayos Zapatillas (Guzmán y Guevara 1998b), pero no se incluyeron los bajos arrecifales profundos ( $>20 \mathrm{~m}$ ) y extensos (ca. $2017 \mathrm{ha}$ ) al norte de éstos. Según Soto et al. (1998), éstas son áreas de alta diversidad e importancia para su conservación, particularmente en macroalgas. El arrecife 43 se describió en representación de estos hábitats (únicamente transectos a esa profundidad), encontrándose una cobertura promedio de corales baja de ca. $10 \%$, y de las más altas coberturas de macroalgas y algas incrustantes con $75 \%$ y $32 \%$, respectivamente (Fig. 4). Se observó un número relativamente alto de corales 
juveniles (ca. 5 individuos $/ \mathrm{m}^{2}$ ), particularmente Siderastrea siderea, Leptoseris cucullata, Favia fragum, Porites astreoides y Agaricia reticulata, en orden de abundancia. Esta última especie se encontró abundantemente aquí en comparación con otras localidades ( Cuadro 2). Posiblemente la densidad alta de reclutas esté asociada a la alta cobertura de algas coralinas y sujetos a una intensa competencia con las abundantes macroalgas. La diversidad de corales es de 23 especies, mientras que la de esponjas encontradas es menos de un tercio de las informadas para la zona (Cuadros 2 y 4 ).

\section{DISCUSIÓN}

Es casi una norma en muchos países la creación de áreas marinas protegidas por presiones socioeconómicas y políticas. En muchos casos los sitios de conservación y el tamaño de éstos no son apropiados y no contemplan la verdadera diversidad marina y el funcionamiento de los ecosistemas de la región. Por otro lado, esto también sucede debido a la falta de información científica disponible a escala local y regional. Para el establecimiento de una área marina protegida (AMP) se deben de considerar ciertos criterios diferentes al de áreas terrestres aunque las metas para su creación sean similares (e.g. transporte y dispersión de larvas, conectividad). Debido a la naturaleza abierta de los ecosistemas marinos. para la protección efectiva de las comunidades y hábitats frágiles se requiere de la creación de áreas más extensas (en comparación a las terrestres), de forma tal que cualquier impacto pueda ser amortiguado y las comunidades críticas permanezcan relativamente intactas. Por lo contrario, existe la tendencia a considerar un factor importante en la selección, diseño y manejo de las AMP el uso continuo que le dan los seres humanos a los recursos dentro y adyacente al área protegida (Kelleher y Kenchington 1991).

El presente estudio define por primera vez para la región de Bocas del Toro, la zona de mayor diversidad de organismos sésiles que son considerados importantes constructores de arrecifes coralinos, y que sin lugar a dudas al- bergan una alta diversidad de plantas y animales asociados, haciendo de estos un ecosistema altamente productivo (Hatcher 1990). Hasta el momento se han recorrido $436 \mathrm{~km}$ de costa y se han descrito 64 arrecifes (Guzmán y Guevara 1989a, b, en prep.) y no se esperaría un aumento considerable en la diversidad de estos organismos, más importante aún, que cambie el centro de diversidad del archipiélago. Lo que es importante aquí es que la escala espacial de estos estudios ha sido sin lugar a dudas un factor importante para la selección de posibles áreas prioritarias de conservación, y además, se establece un precedente a nivel nacional para estudios sistemáticos e integrales de la biodiversidad marina.

El número de especies de corales escleractínidos para todo el archipiélago de Bocas del Toro aumentó en $5.5 \%$ con el registro de tres nuevas especies, Colpophyllia amaranthus, Manicina mayori y Mycetophyllia reesi, es decir a 57 en total. Las dos primeras son conocidas de otras áreas de Panamá pero sse informan por primera vez (ver Holst y Guzmán 1993), aumentando así a un total de 64 especies para el país, incluyendo corales escleractínidos e hidrocorales. Esto implica que en el archipiélago se encuentra el $89 \%$ de las especies del Caribe panameño y el $96 \%$ o más de éstas (incluyendo dos especies en Guzmán y Guevara 1998b), están distribuidas en los arrecifes de la denominada "zona de alta diversidad" encontrada en el presente estudio (Fig. 1, zona amarilla). De igual forma, la diversidad de octocorales aumentó a 29 especies (16\%) con la adición de cuatro nuevos registros para Bocas del Toro; Plexaura kuna, Eunicea tourneforti, Muricea pinnata y Pterogorgia guadalupensis. Las esponjas fue el taxón que mayor cambio experimentó, con un incremento de 16 nuevas especies (30\%) y un total de 53 para el archipiélago. Se encuentran en la "zona de alta diversidad" el $94 \%$ y $97 \%$ de las esponjas y octocorales, respectivamente (se incluyen dos especies de octocorales informadas para Cayos Zapatillas y lado este de Isla Bastimentos; Guzmán y Guevara 1998b). En resumen, se podría decir que en promedio el $95.7 \%$ de la 
diversidad (corales, octocorales y esponjas) se encuentra en esa zona.

En ambientes terrestres se ha criticado duramente el uso de la abundancia y riqueza de especies como indicadores de sitios para la conservación, ya que no necesariamente coinciden especies raras y endemismo con alta riqueza de especies. Pero, en la mayoría de los casos se ha coincidido en que la escala de muestreo juega un papel muy importante (grano grueso o fino, local o regional), ya que ésta permite que en las áreas protegidas se manifiesten los procesos que afectan el funcionamiento de comunidades y ecosistemas (Prendergast et al. 1993, Lombard 1995, Conroy y Noon 1996, Kerr 1996). Existe un patrón consistente que sugiere que los límites del área protegida de Isla Bastimento deben ser modificados, ya que no se incluye la mayor diversidad de organismos constructores de arrecifes. Es importante tomar en consideración que el centro de alta diversidad descrito anteriormente contiene además, especies raras y poco abundantes con respecto a otras localidades del Gran Caribe. Por ejemplo, los corales acropóridos Acropora palmata y A. cervicornis se consideraban entre las principales especies constructoras de arrecifes, y actualmente considerados por la comunidad científica internacional para incluirlas en la lista de especies amenazadas, debido a que su abundancia ha disminuido notablemente durante las dos últimas décadas o han desaparecido de ciertas localidades (Gladfelter 1982). El sector del archipiélago con mayor abundancia de acropóridos se encuentra dentro de esta área de alta diversidad (Guzmán y Guevara 1998b, presente estudio). Por lo tanto en este caso, el argumento no se basa exclusivamente en preservar la diversidad, sino también hábitats importantes para toda la región, lo que es recomendable en un manejo integral de la zona costera para mantener los diversos procesos que moldean un ecosistema y sus pesquerías (Allison et al. 1998, Done y Reichelt 1998). Lo anterior demuestra en parte, que algunos de los principios para la conservación de ambientes terrestres no son aplicables a ecosistemas marinos, aunque si coinciden en la necesidad de una escala mayor.

Con respecto a la escala espacial, es notable que por nuestros resultados se deberían considerar algunos cambios en el área protegida actual, ya que se permitiría obtener una mayor regeneración de poblaciones afectadas naturalmente o por el hombre, a nivel local (Bocas del Toro), sub-regional (Panamá) y regional (Centroamérica y el Caribe). Se ha demostrado que existe una alto grado de dependencia entre la riqueza de especies a estas escalas y se estima que las comunidades arrecifales locales se enriquecen de fuentes regionales (sub-regionales?) de especies (Karlson y Cornell 1999). Sin embargo, este enriquecimiento de especies varía geográficamente como se esperaría, y contrariamente a lo expuesto anteriormente en este párrafo, para la región del Caribe (Atlántico Oeste) se considera que estos procesos son menos sensitivos a variar en gran escala, ya que la diversidad es relativamente homogénea (sensu Karlson y Cornell 1998, 1999). Esto podría cuestionarse, ya que desconocemos los fenómenos a larga escala que afectan nuestra región (e.g. Centroamérica).

Llama la atención la alta diversidad encontrada, considerando la calidad del agua que limitan la estructura y desarrollo de los arrecifes del archipiélago, y cuyo nivel es inferior a los de otras áreas, como San Blas por ejemplo (Guzmán y Guevara 1998a). Por lo general y dependiendo de la profundidad, son alrededor de tres las especies dominantes y principales constructoras de arrecifes en Bocas del Toro (e.g. Porites furcata, Agaricia tenuifolia, Siderastrea siderea) (Guzmán y Guevara 1998a, b). Es notable que la alta escorrentía y sedimentación, la falta de transparencia del agua en los dos primeros metros de profundidad y la alta exposición al oleaje, limitan el desarrollo, la diversidad y la zonación vertical de especies en los arrecifes internos y externos del archipiélago (Guzmán y Guevara 1998a, b), como se ha observado en otros arrecifes del Caribe (Cortés y Risk 1985, Tomascik y Sanders 1987, Acevedo et al. 1989, Rogers 1990). Sin embargo, la zona descrita se encuentra en un enclave semi-protegido por la orografía natural, que parece responder a un 
flujo alto de corrientes marinas que convergen de mar abierto y desde el interior de la Laguna de Chiriquí, lo cual permitiría un mayor intercambio larval entre poblaciones de afuera del achipiélago (regional) y locales. Esto y el problema de escala expuesto al final del párrafo anterior podría responderse con más estudios que contemplen el transporte de masas de aguas costeras y genética de poblaciones.

Finalmente, quisiéramos mencionar el extenso banco arrecifal ubicado al sur de Cayo Aggua y dentro de Laguna de Chiriquí (Fig. 1), que sirve para ilustrar la vulnerabilidad de los arrecifes a efectos naturales tales como el blanqueamiento y muerte de corales asociado al calentamiento del mar (Goreau y Hayes 1994, Wilkinson et al. 1999), y que podrían deteriorarse con la actual expansión de asentamientos indígenas, desarrollo turístico y deforestación. El nivel de desarrollo observado en esos arrecifes hasta $25 \mathrm{~m}$ de profundidad es de los mejores para el archipiélago. Sin embargo, encontramos arrecifes prácticamente muertos y formado por especies masivas importantes, muertas, en posición vital a partir de los $4 \mathrm{~m}$ (cobertura promedio de $17 \%$ ). El flujo constante de corrientes proveniente de mar abierto a través del canal formado entre Isla Popa y Cayo Agua, donde se observa gran abundancia y diversidad de especies masivas constructoras de arrecifes y observadas muertas en el bajo, e.g., Montastraea annularis, $M$. faveolata, $M$. franksi, Siderastrea siderea y otras, es importante para el restablecimiento de este banco arrecifal. Según relatan los pescadores del sector, este fue un sitio que albergaba una de las mejores pesquerías del archipiélago pero desapareció en 1970 después de las torrenciales lluvias de abril $(614.4 \mathrm{~mm})$ y diciembre (1 012.2 $\mathrm{mm}$ ) (total de $3702.8 \mathrm{~mm}$ ), las más intensas que se conozcan en la zona para este siglo (Bocas Fruit Co., Finca 8, Changuinola; C.S. Stephens, com. per.). Semanas de alta sedimentación provocada por las plumas de los ríos desbordados al sur y suroeste de la Laguna de Chiriquí enterraron estos arrecifes. Sin embargo, el flujo de agua mencionado anteriormente pareciera haber removido estos sedimentos y es- tá permitiendo la recuperación lenta de los arrecifes. Actualmente se observan densidades de seis reclutas de corales $/ \mathrm{m}^{2}$, y se podría especular que la pesquería en el lugar podría mejorar en los próximos años, asumiendo que el ecosistema esté funcional y la tasa de sedimentación no aumente (sensu Wittenberg y Hunter 1992, Hughes 1994). No obstante se necesita proteger los ecosistemas terrestres de la islas aledañas y en tierra firme para evitar que continúe el deterioro.

\section{RECOMENDACIONES}

El presente estudio demuestra la presencia de un centro de alta diversidad, no descrito anteriormente para el archipiélago, y localizado en el sector semi-expuesto comprendido por Isla Bastimentos-Cayos Zapatillas-Isla PopaCayo Agua. Cinco recomendaciones pueden ofrecerse a partir de nuestros resultados:

1. Modificar los límites existentes del Parque Nacional Marino Isla Bastimentos (PNMIB), de forma que se protejan las áreas que albergan la mayor diversidad y la mayor abundancia de especies amenazadas. En particular se sugiere el sitio arriba descrito (Fig. 1), pero además, deben de incluirse otros arrecifes al sur de Isla Colón, oeste de Isla Cristóbal y alrededores de Isla Pastores (Guzmán y Guevara 1998b). Esto implica un cambio notable en el sentido legal y social. Se deben de ensayar varios modelos de áreas protegidas para el archipiélago de Bocas del Toro, que incluyan el establecimiento de un nuevo parque nacional con límites modificados o de varias reservas de tamaño pequeño interconectadas, es decir, una red de áreas protegidas. $\mathrm{Al}$ respecto, es necesario realizar una consulta general que involucre a los principales actores de la sociedad civil y entidades del estado panameño. Cualquier cambio que se desee deberá estar acompañado de una efectiva implementación de las leyes y de 
mejorías en la educación ambiental y percepción de los pobladores hacia nuevos modelos de protección (sensu Alder 1996).

2. Incorporar los resultados del presente estudio en la elaboración del primer plan de manejo para el PNMIB, y se de comienzo al período de consultas sugerido en el punto anterior. El PNMIB no ha tenido ningún plan de manejo desde su creación en diciembre de 1986.

3. Continuar, reforzar y ampliar a otras localidades la actual campaña de educación ambiental, ya que esta ayudaría en el proceso de transformación y selección de áreas protegidas. Esta campaña debe integrar de cierta forma los objetivos políticos y criterios socioeconómicos a largo plazo (sensu Lockwood et al. 1997). Definitivamente la campaña en Isla Popa y Cayo Agua es de vital importancia.

4. Detener o regular apropiadamente el uso actual de las tierras adyacentes a la zona costera, de forma tal que el desarrollo turístico, la agroforestería y la ganadería, no tengan impactos a largo plazo sobre los ecosistemas marinos, como se está observando en muchos lugares del archipiélago. Se debe responder a un plan regional de desarrollo consensuado, donde los pobladores deben ayudar en la protección de los bosques y de la pesca en sus propias islas.

5. Controlar el turismo de buceo existente dentro del área señalada como "zona de alta diversidad", ya que es aquí donde se observa una mayor actividad por parte de los operarios de turismo. Se deben de promulgar medidas inmediatas que regulen el uso de estos arrecifes y ayuden a mitigar el impacto comprobado de esta actividad sobre el ecosistema (sensu Allison 1996, Harriott et al. 1997, Rouphael y Inglis 1997), las cuales deben de incluir la educación a los operarios y turistas (Davis y Tisdell 1996, Medio et al. 1996).

\section{AGRADECIMIENTOS}

Agradecemos a A. González y al personal de la Fundación para la Protección del Mar (PROMAR) en Bocas del Toro y Panamá por el apoyo brindado durante este estudio. Agradecemos a K. Kauffman y J.B. Del Rosario por preparar las estadísticas descriptivas. A J. Cortés por su extensa revisión al manuscrito. Este estudio fue financiado por la Fundación Natura-FIDECO (Proyecto P4-98002-PROMAR), PROMAR y el Smithsonian Tropical Research Institute.

\section{RESUMEN}

En estudios anteriores se encontró que el área protegida del Parque Nacional Marino de Isla Bastimentos (PNMIB) no albergaba arrecifes en buen estado de conservación, desarrollo y diversidad, recomendándose ampliar el reconocimiento de arrecifes a otras áreas del archipiélago de Bocas del Toro, en particular el sector oriental del parque marino. En el presente estudio, se hizo un reconocimiento - ca. $107.5 \mathrm{~km}$ en total - detallado de la zona costera insular de islas Pastores, Cristóbal, Popa y Cayo Agua, donde se evaluó la distribución de los arrecifes coralinos. Se describieron un total de 24 arrecifes, los cuales representan las formaciones típicas para las distintas localidades. Se define por primera vez para el archipiélago el centro de mayor diversidad de organismos sésiles, que son importantes constructores de arrecies coralinos. El número de especies de corales escleractínidos para todo el archipiélago de Bocas del Toro aumentó a 57 y se actualizan también los registros para Panamá aumentando así a un total de 64 especies para el país. Esto implica que el archipiélago alberga el $89 \%$ de las especies de Panamá, de las cuales $96 \%$ o más están distribuidas en los arrecifes de la "zona de alta diversidad" encontrada en el presente estudio. De igual forma, la diversidad de octocorales aumentó a 29 especies $(16 \%)$ y las esponjas experimentaron un incremento de 16 especies $(30 \%)$ para un total de 53 especies. Se encuentran en la "zona de lata diversidad" el $94 \%$ y $97 \%$ de las especies de esponjas y octocorales, respectivamente. Se presentan cinco recomendaciones generales, siendo las más importante, modificar los límites existentes del PNMIB, de forma que se prote jan las áreas que albergan la mayor diversidad general y la mayor abundancia de especies amenazada, o crear una red de áreas protegidas dentro del archipiélago. 


\section{REFERENCIAS}

Acevedo, R., J. Morelock \& R.A. Olivieri. 1989. Modification of coral reef zonation by terrigenous sediment stress. Palaios 4: 92-100.

Alder, J. 1996. Costs and effectiveness of education and enforcement, Cairns section of the Greatbarrier Reef Marine Park. Environm. Manag. 20: 541-551.

Allison, W.R. 1996. Snorkeler damage to reef corals in the Maldive Islands. Coral Reefs 15: 15-18.

Allison, W.R., J. Lubchenco \& M.H. Carr. 1998. Marine reserves are necessary but not sufficient for marine conservation. Ecol. Applic. 8: S79-S92.

Alvarado, R.H. 1987. Estudio preliminar para el establecimiento del Parque Nacional Marino Isla Bastimentos. INRENARE-CATIE-ANCON, Panamá. $11 \mathrm{p}$

Anónimo. 1988. Atlas Nacional de la República de Panamá. Instituto Geográfico Nacional "Tommy Guardia”, Panamá. 222 p.

Bryant, D., L. Burke, J. McManus \& M. Spalding. 1998. Reefs at Risk: A map-based indicators of threats to the world's coral reefs. World Resources Institute, Washington, D.C. $56 \mathrm{p}$.

Coates, A.G. \& J.B.C. Jackson. 1998. Neogene sedimentary basins of Costa Rica and Panama. 11 th Conference Inter. Bryozoology Association, Panama. 39 p.

Collins, L.S., A.F. Budd \& A.G. Coates. 1996. Earliest evolution associated with closure of the Tropical American Seaway. Proc. Nat. Acad. Sci. 93: 6069-6072.

Conroy, M.J. \& B.R. Noon. 1996. Mapping of species richness forconservation of biological diversity: conceptual and methodological issues. Ecological Applications 6:763-773.

Cortés, J. \& M.J. Risk. 1985. A reef under siltation stress: Cahuita, Costa Rica. Bull. Mar. Sci. 36 339-356.

Davis, D. \& C. Tisdell. 1996. Economic management of recreational scuba diving and the environment. J. Environm. Manag. 48: 229-248.

Done, T.J. \& R.E. Reichelt. 1998. Integrated coastal zone and fisheries ecosystem management: generic goals and performance indices. Ecol. Applic. 8: S110-S118.
Gladfelter, W.B. 1982. White-band disease in Acropora palmata: implications for the structure and growth of shallow reefs. Bull. Mar. Sci. 32: 639-643.

Greb, L., B. Saric, H. Seyfried, T. Broszonn, S. Brauch, G. Gugau, C. Wiltschko \& R. Leinfelder. 1996 Ökologie ind sedimentologie eines rezenten rampensystem an der Karibikküste von Panama. Profil, Band 10, Universität Stuttgart, 168 p.

Gordon, B.L. 1982. A Panama forest and shore: natural history and Amerindian culture in Bocas del Toro. Boxproof, California. $178 \mathrm{p}$

Goreau, T.J. \& R.L. Hayes.1994. Coral bleaching and ocean "hot spots". Ambio 23: 176-180.

Guzmán, H.M. \& C. Guevara. 1998a. Arrecifes coralinos de Bocas del Toro, Panamá: I. Distribución, estructura y estado de conservación de los arrecifes continentales de la laguna de Chiriquí y la Bahía Almirante. Rev. Biol. Trop. 46: 601623.

Guzmán, H.M. \& C. Guevara. 1998b. Arrecifes coralinos de Bocas del Toro, Panamá: II. Distribución, estructura y estado de conservación de los arrecifes de las islas Bastimentos, Solarte, Carenero y Colón. Rev. Biol. Trop. 46: 893-916.

Harriott, V.J., D. Davis \& S.A. Banks. 1997. Recreational diving and its impact in marine protected areas in eastern Australia. Ambio 26: 173-179.

Hatcher, B.G. 1990. Coral reefs primary productivity: a hierarchy of pattern and process. TREE 5: 149-155

Holst, I. \& H.M. Guzmán. 1993. Lista de corales hermatípicos (Anthozoa: Scleractinia; Hydrozoa: Milleporina) a ambos lados del istino úc Panañiá Rev. Biol. Trop. 41: 535-540.

Hughes, T.P. 1994. Catastrophes, phase shifts, and largescale degradation of a Caribbean coral reef Science 265: 1547-1551

Karlson, R.H \& H.V Cornell. 1998. Scale-dependent variation in local vs. regional effects on coral species richness. Ecol. Monog. 68: 259-274.

Karlson, R.H \& H.V Cornell. 1999. Integration of local and regional perspectives on the species richness of coral assemblages. Amer. Zool. 39: 104-112.

Kelleher, G. \& R. Kenchington. 1991. Guidelines for establishing marine protected areas. IUCN, Gland, Suiza. 79 p. 
Kerr, J.T. 1996. Species richness, endemism, and the choice of areas for conservation. Conserv. Biol. 11: 1094-1100.

Lockwood, M., D.G. Boss \& G. Glazebrook. 1997. Integrated protected area selection in Australian biogeograpic regions. Environm. Manag. 21: 395-404.

Lombard, A.T. 1995. The problems with multi-species conservation: do hotspots, ideal reserves and existing reserves coincide? S. Afr. J. Zool. 30: 145-163.

Loya, Y. 1972. Comnunity structure and species diversity of hermatypic corals at Eilat, Red Sea. Mar. Biol. 13: 100-123.

Medio, D., R.F.G. Ormond \& M. Pearson. 1997. Effect of briefings on rates of damage to corals by scuba diving. Biol. Conserv. 79: 91-95.

Prendergast, J.R., R.M. Quinn, J.H. Lawton, B.C. Eversham \& D.W. Gibbons. 1993. Rare species, the coincidence of diversity hotspots and conservation strategies. Nature 365: 335-337.

Rodríguez, E., R. Almanza \& R. Alvarado. 1993. Situación biofísica y ambiental de la Provincia de Bocas del Toro, p. 55-72. In S. Heckadon-Moreno (ed.). Agenda Ecológica y Social para Bocas del Toro. Continental, Panamá

Rogers, C.S.1990. Responses of coral reefs and reef organisms to sedimentation. Mar. Ecol. Prog. Ser. 62: $185-202$.

Rouphael, A.B. \& G.J. Inglis.1997. Impacts of recreational scuba diving at sites with different reef topographies. Biol. Conserv. 8: 39-336.
Soto, R., H.M. Guzmán, Z. Pinzón, J. Moreno, C. Gamboa, M. Montoya \& R. Vargas. 1998. Evaluación ecológica rápida del Parque Nacional Marino Isla Bastimentos y áreas adyacentes, provincia de Bocas del Toro. Tomo 3: recursos costeros-marinos. ANCON, Panamá. 248 p.

Sullivan, K.M. \& M. Chiappone. 1992. A comparison of belt quadrat and species presence/absence sampling of stony corals (Scleractinia and Milleporina) and sponges for evaluating species patterning on patch reefs of the central Bahamas. Bull. Mar. Sci. 50: 464-488.

Sullivan, K.M., M. Chiappone \& C. Lott. 1994. Abundance patterns of stony corals on platform margin reefs of the Caicos Bank. Bahamas $\mathbf{J}$. Sci. 1: 2-12.

Tomascik, T. \& F. Sanders. 1987. Effects of eutrophication on reef-building corals II. Structure of scleractinians coral communities on fringing reefs, Barbados, West Indies. Mar. Biol. 94: 53-75.

Wilkinson, C., O. Linden, H. Cesar, G. Hogson, J. Rubens and A.E. Strong. 1999. Ecological and socioeconomic impacts of 1998 coral mortality in the Indian Ocean: an ENSO impact and a warning of future change? AMBIO 28: 182-196.

Wittenberg, M. \& W. Hunter. 1992. Effects of eutrophication and sedimentation on juvenile corals. Mar. Biol. 112: 131-138. 